\title{
Augmentative and Alternative Communication
}

\section{Revealing Personhood Through Biomusic of Individuals Without Communicative Interaction Ability}

\section{Stefanie Blain \& Patricia McKeever}

To cite this article: Stefanie Blain \& Patricia McKeever (2011) Revealing Personhood Through Biomusic of Individuals Without Communicative Interaction Ability, Augmentative and Alternative Communication, 27:1, 1-4, DOI: 10.3109/07434618.2011.556663

To link to this article: https://doi.org/10.3109/07434618.2011.556663

曲 Published online: 01 Mar 2011.

Submit your article to this journal $\widetilde{ }$

III Article views: 246

Q View related articles $\asymp$

Citing articles: 5 View citing articles $\longleftarrow$ 


\title{
Forum Note
}

\section{Revealing Personhood Through Biomusic of Individuals Without Communicative Interaction Ability}

\author{
STEFANIE BLAIN* and PATRICIA McKEEVER \\ University of Toronto and Bloorview Research Institute, Toronto, Ontario, Canada
}

\begin{abstract}
An increasing number of people are unable to engage in communicative interactions because of their inability to move, speak, and control traditional augmentative and alternative communication devices. According to social interactionist theory, the existence of the human self hinges on successful interaction with others; consequently, those who cannot interact are at risk of not being accorded personhood. This article is a request for research towards the development of technologies that would give caregivers access to the autonomic physiological signals of individuals who are unable to communicate. It explores the hypothesis that meaningful manifestation of signals that change according to mental and emotional states will reveal individual personhood. We hypothesize that music is ideally suited as the basis of interaction with this population, and that physiologically driven musical expression is a form of individual biomusic that can enrich social interaction.
\end{abstract}

Keywords: Communication; Personhood; Music; Physiological signals

\section{INTRODUCTION}

Advanced medical technologies have led to an increased prevalence of people whose survival depends entirely on respiratory and nutrition technology and multiple human caregivers (Carnevale, Rehm, Kirk, \& McKeever, 2008). Many of these individuals have minimal or no ability to move or speak, and do not have a reliable means of accessing traditional augmentative and alternative communication (AAC) devices, computers, or assistive technologies. Thus, even those with high cognitive abilities (e.g., people with locked-in syndrome) are not able to engage in communicative interactions. The responsibilities and implications of supporting this population are only just being realized. Improvements in medical technology over the last 20 years have, in effect, created "a new strain of human beings" (Brown, 2009, p. 96) who require an unprecedented level of care. Despite the extraordinary physical, psychological, social, and financial challenges involved in supporting these persons, most are cherished by their families, regardless of whether they are being cared for at home or in long-term care settings (Carnevale et al., 2008). However, the fact that they are unable to engage in communicative interactions places substantial strain on family members and other caregivers. Caregivers perceive communication to be unidirectional and thus can experience considerable distress and frustration when attempting to interact with individuals who cannot produce vocal or other observable responses (Costello, 2000; Happ, 2001). When social interaction is unreciprocated, caregivers often confine their interactions to nursing, therapies, or custodial care. At a time when ethical debates regarding the growing population of profoundly disabled individuals are gathering pace, it is important to reflect upon and examine our response to these most vulnerable members of our society. We propose an intervention that may help those who seem unable to communicate their personhood and may enable others to respond to them in an individualized manner: the generation of music from autonomic physiological signals.

\footnotetext{
*Corresponding author. C640 Med Inn Building, 1500 East Medical Center Drive, Ann Arbor, MI 48109-5841G, USA. E-mail: sblain@umich.edu
} 
According to social interactionist theory, the existence of the human self hinges on successful interaction with others (Cooley, 1972; Mead, 1970); those who cannot engage in this way are at risk of not being accorded personhood by others. The concept of personhood is mainly found in discourses on transcendence, ethics, and social psychology; it is defined here as "a standing or status that is bestowed upon one human being by others, in the context of relationship and social being" (Kitwood, 1997, p. 8). Feeling, emotions, and the ability to live in relationships are linked far more strongly to the concept of personhood than autonomy and rational capability (Post, 1995). The accordance of personhood, then, is predominantly dependent on the ability to express these attributes, which is very difficult for a person who cannot engage in communicative interactions. In essence, he or she is not perceived as an "other" with whom interaction is possible because of the inability to demonstrate putative cardinal human attributes of co-presence and reciprocity (Waksler, 2006). In the absence of embodied and socially interconnected manifestations of self, perceiving the personhood of these individuals is fraught with challenges and may not be accomplished (Kontos, 2005). This has significant ramifications for the quality of life of such persons and those who care for them.

To date, only a few interventions have addressed issues of quality of life and quality of support for people who are unable to engage in communicative interactions. These interventions have predominantly consisted of either presenting preferred items, stimuli, or activities to increase indicators of happiness; or training direct support staff in techniques to increase the quality and quantity of interactions with those for whom they are caring (Maes, Lambrechts, Hostyn, \& Petry, 2007). Considering the concept of personhood in relational terms, these interventions are promising, in theory; however, the majority of studies report only moderately positive effects of these interventions on the behavior of caregivers and/or care recipients (Maes et al., 2007). This is likely because individuals with very limited expressive repertoires are usually also not able to express themselves via gestures or other physical behaviors. Thus, we look towards physiological rather than physical responses to enable interaction with others.

It is well known that physiological signal changes in the autonomic nervous system (ANS) are connected to emotional states (Bauer, 1998; Ekman, Levenson, \& Friesen, 1983), and much work has been conducted to classify emotion according to patterns in autonomic physiological signals (Cacioppo \& Tassinary, 1990; Picard,
Vyzas, \& Healey, 2001). We conjecture that enabling caregivers to access the autonomic physiological signals of those unable to engage in communicative interactions may facilitate the perception of their personhood. Furthermore, we hypothesize that the universal medium of music is ideally suited to form the basis of meaningful interaction with this oft-perceived "silent" population.

Music seems to be an optimal medium for communicating personhood, since musical sounds and rhythms constitute a universal, nonverbal signifying system capable of expressing human emotion. While the emotional content of music is acknowledged by many music scholars, some posit that music can create an emotional geography of relatedness between individuals (Wood, 2002). It has been argued that "music - and our responses to it - is an expression of emotions and drives that have the potential to recreate our social and spatial selves. These qualities are what make music about being and becoming, and to get to grips with this is to understand the way identities are made" (Wood, Duffy, \& Smith, 2007, p. 883). In the time-space where music is produced and heard, the intimate emotional quality of human relations is laid bare. Performing music, therefore, is about intimate encounters with others, enabling participants to connect emotionally and to share a dimension of human experience beyond language, movement, or gesture. Drawing from the body of literature that connects changes in autonomic physiological signals of the human body to emotional state, the potential for physiologically generated music to convey an individual's personhood becomes clear. The autonomic nervous system in individuals unable to engage in communicative interaction often remains intact despite damage to the somatic nervous system (Doble, Haig, Anderson, \& Katz, 2003); in harnessing and manifesting changes in these signals through music, they may express the co-presence and reciprocity required for others to perceive them as responsive and available for interaction. We believe that physiologically driven musical expression can create unique biomusic for each individual, which changes dynamically in response to the individual's emotions and reactions to their environment, creating the basis for meaningful interaction with others.

Autonomic physiological signals have been used to generate music for different purposes. Clinically, physiological signals and sound have been combined for the purposes of sonification, an auditory means of conveying and perceiving information. Sonification of electroencephalography (EEG) signals, for example, has been useful 
for fast screening of long-term EEG recordings and real-time monitoring of EEG recording sessions (de Campo, Hoeldrich, \& Eckel, 2007). Artistically, physiological signals and sound have been combined to create interactive art performances that are based upon the physiological state of performers (Brouse et al., 2006). Arslan et al. (2005) and Brouse et al. (2006) provide a full review of both biosignal sonification and physiologically driven musical interfaces. However, the potential of combining the ability to recognize emotions from patterns of ANS signals and the capacity for music to emotionally connect two individuals remains largely untapped. It is not difficult to envision a future technology that discerns emotions from an individual's ANS patterns and intelligently combines elements of pitch, rhythm, harmony, mode, tempo, dynamic, and timbre to musically convey this precise emotional state. While emotion recognition and music psychology research are not yet sufficiently developed to achieve this vision, many meaningful intermediate steps exist in the research towards this goal. For example, electrodermal reactions (EDRs) are an established indicator of ANS arousal levels. Musical mappings that make EDRs explicit (e.g., mapping pitch to the absolute value of the electrodermal activity, thereby depicting EDRs as a fast, upwards glissando) could convey the dynamic internal state of an individual without communicative interaction ability. These reactions may occur, for example, as such an individual witnesses a loved one appear, and the co-presence and reciprocity revealed by his or her changing biomusic has the potential to form the basis of interactions that establish personhood.

Significant ethical issues must be considered throughout the research and development of biomusic for individuals unable to engage in communicative interactions. As music enables an individual to articulate a fluid form of subjectivity, it leaves room for others to interpret its meaning. Researchers must acknowledge both the risk of listeners projecting false meaning upon an individual's biomusic and the subtle boundary between "giving a voice" and "interpreting a voice." It is crucial that the technical development of this idea be done in dialogue with all caregivers involved with these individuals so that the limits for interpretation of the biomusic can be knowledgeably set and enforced. Within these ethical considerations, the growth of a knowledge base necessary for the technical development of this idea will be driven by several fundamental research questions: Does exposure to the dynamic physiological states of individuals without communicative interaction ability augment the ability for caregivers to perceive the personhood of these individuals? What combinations of musical elements and physiological signals are most effective in conveying an individual's responsiveness and emotional state? How can these combinations of elements be given the qualities of music and which are best at creating an emotional geography of relatedness between individuals? How do we build physiological, signal-based emotion-recognition algorithms for individuals who do not have interactive communicative abilities and therefore are also unable to report their thoughts or feelings? These questions are inherently interdisciplinary in nature, and, in order to be addressed in any meaningful sense, will require the efforts of multidisciplinary teams and the development of research methods that transcend current disciplinary confines.

While the proposed research is fraught with technical and ethical challenges, our purpose here is not to explore potential solutions, but to draw attention to the possibility for inclusion in the human community through the presentation of the biomusic of those without communicative interaction ability. We believe this biomusic has the potential to modify the environmental milieu and sound spaces of these apparently silent individuals and to enable others to recognize their embodied personhood. We strongly advocate for research towards this goal in the spirit of enriching interpersonal interactions with individuals with all levels of communicative ability and creating spaces of community and belonging.

\section{Author Note}

The work for the manuscript was conducted in affiliation with the University of Toronto and Bloorview Kids Rehab. The corresponding author is now completing a postdoctoral fellowship at the University of Michigan.

The authors are indebted to Shauna Kingsnorth and Elaine Biddiss for their contributions to the development of the ideas in this manuscript.

Declaration of interest: The authors report no conflict of interest. The authors alone are responsible for the content and writing of the paper.

\section{References}

Arslan, B., Brouse, A., Castet, J., Filatriau, J. J., Lehembre, R., Noirhomme, Q., \& Simon, C. (2005). Biologicallydriven musical instruments. Proceedings of the 1st Summer Workshop on Multimodalinterfaces (eNTERFACE-05), Mons, Belgium (pp. 21-33). 
Bauer, R. M. (1998). Physiologic measures of emotion. Journal of Clinical Neurophysiology, 15, 388-396.

Brouse, A., Filatriau, J. J., Gaitanis, K., Lehembre, R., Macq, B., Miranda, E., \& Zenon, A. (2006). An instrument of sound and visual creation driven by biological signals. Proceedings of the 2nd Summer Workshop on Multimodal Interfaces (eNTERFACE'06), Dubrovnik, Croatia (pp. 59-68).

Brown, I. (2009). The boy in the moon: A father's search for his disabled son. Toronto: Random House Canada.

Cacioppo, J. T., \& Tassinary, L. G. (1990). Inferring psychological significance from physiological signals. American Psychologist, 45(1), 16-28.

Carnevale, F. C., Rehm, R. S., Kirk, S., \& McKeever, P. (2008). What we know (and do not know) about raising children with complex continuing care needs. Journal of Child Health Care, 12(1), 4-6.

Cooley, C. H. (1972). The looking glass self. In J. Manis \& B. Meltzer (Eds.), Symbolic interaction: A reader in social psychology (pp. 231-233). Boston, MA: Allyn \& Bacon.

Costello, J. M. (2000). AAC intervention in the intensive care unit: The Children's Hospital Boston model. Augmentative \& Alternative Communication, 16, 137-153.

de Campo, A., Hoeldrich, R., \& Eckel, G. (2007). New sonification tools for EEG data screening and monitoring. In Proceedings of the 13th International Conference on Auditory Display, Montreal, Canada (pp. 536-542).

Doble, J. E., Haig, A. J., Anderson, C., \& Katz, R. (2003). Impairment, activity, participation, life satisfaction, and survival in persons with locked-in syndrome for over a decade: Follow-up on a previously reported cohort. The Journal of Head Trauma Rehabilitation, 18, 435-444.

Ekman, P., Levenson, R. W., \& Friesen, W. V. (1983). Autonomic nervous system activity distinguishes among emotions. Science, 221(4616), 1208-1210.
Happ, M. B. (2001). Communication with mechanically ventilated patients: State of the science. AACN Clinical Issues, 12, 247-258.

Kitwood, T. (1997). Dementia reconsidered: The person comes first. Buckingham: Open University Press.

Kontos, P. C. (2005). Embodied selfhood in Alzheimer's disease: Rethinking person-centred care. Dementia, 4(4), 553-570.

Maes, B., Lambrechts, G., Hostyn, I., \& Petry, K. (2007). Quality-enhancing interventions for people with profound intellectual and multiple disabilities: A review of the empirical research literature. Journal of Intellectual \& Developmental Disability, 32(3), 163-178.

Mead, G. H. (1970). Development of the self through play and games. In G. P. Stone \& H. A. Farberman (Eds.), Social psychology through symbolic interaction (pp. 537545). London: Ginn-Blaisdell.

Picard, R. W., Vyzas, E., \& Healey, J. (2001). Toward machine emotional intelligence: Analysis of affective physiological state. IEEE Transactions on Pattern Analysis and Machine Intelligence, 23, 1175-1191.

Post, S. G. (1995). The moral challenge of Alzheimer disease (1st edn). Baltimore, MD: John Hopkins University Press.

Waksler, F. C. (2006). Analogues of ourselves: Who counts as an other? Human Studies, 28, 417-429.

Wood, N. (2002). 'Once more with feeling': Putting emotion into geographies of music. In L. Bondi, H. Avis, R. Bankey, A. Bingley, J. Davidson, R. Duffy, V. I. Einagel, ... N. Wood (Eds.), Subjectivities, knowledges and feminist geographies (pp. 57-71). Lanham, MD: Rowman and Littlefield.

Wood, N., Duffy, M., \& Smith, S. (2007). The art of doing (geographies of) music. Environment and Planning D: Society and Space, 25, 867-889. 